\title{
Main Injector Particle Production Experiment (MIPP) at Fermilab
}

\author{
Sonam Mahajan \\ Department of Physics, Panjab University, Chandigarh-160014, India \\ Fermi National Accelerator Laboratory, Batavia-60510, Illinois, USA \\ (For the MIPP Collaboration) \\ (sonam@fnal.gov)
}

\begin{abstract}
The Main Injector Particle Production Experiment at Fermilab uses particle beams of charged pions, kaons, proton and anti-proton with beam momenta of 5 to $90 \mathrm{GeV} / \mathrm{c}$ and thin targets spanning the periodic table from (liquid) hydrogen to uranium to measure particle production cross sections in a full acceptance spectrometer with charged particle identification for particles from 0.1 to $120 \mathrm{GeV} / \mathrm{c}$ using Time Projection Chamber, Time of Flight, multicell Cherenkov, and Ring Imaging Cherenkov detectors and Calorimeter for neutrons. Particle production using $120 \mathrm{GeV} / \mathrm{c}$ protons from Main Injector on the MINOS target was also measured. We describe the physics motivation to perform such cross section measurements and highlight the impact of hadronic interaction data on neutrino physics. Recent results on forward neutron cross sections and analysis of MINOS target data are also presented.
\end{abstract}

Keywords: MIPP; Neutrinos; Neutrons; NuMI

PACS: $13.60 . \mathrm{Hb}, 13.85 . \mathrm{Ni}, 13.60 . \mathrm{Rj}, 13.75 . \mathrm{Cs}, 25.40 . \mathrm{Ep}$, 14.60.Lm

\section{INTRODUCTION}

The MIPP is a hadron production experiment which uses $120 \mathrm{GeV} / \mathrm{c}$ Main Injector primary protons to produce secondary beams of $\pi^{ \pm}, \mathrm{K}^{ \pm}, \mathrm{p}$ and $\overline{\mathrm{p}}$ from $5 \mathrm{GeV} / \mathrm{c}$ to $90 \mathrm{GeV} / \mathrm{c}$. It was designed to measure the total charged particle production of $\pi^{ \pm}, \mathrm{K}^{ \pm}, \mathrm{p}$ and $\overline{\mathrm{p}}$ using beams of charged pions, kaons, proton and anti-proton on nuclear targets. The MIPP measures particle production crosssections off various nuclei including hydrogen, MINOS target and thin targets of Beryllium, Carbon, Bismuth and Uranium.

The MIPP is a full acceptance spectrometer. The layout of the apparatus is shown in Figure 1. It provides data to reconstruct incoming beam particles and secondary particles from interactions on the target. The three drift chambers upstream of the target (BCs) are able to reconstruct the incident particle trajectory (track). The Time Projection Chamber (TPC), four drift chambers (DCs), and two propotional wire chambers (PWCs) are used to reconstruct tracks coming out of an interaction.

The MIPP provides excellent particle ID (PID) separation. It uses two differential beam Cherenkov counters for identifying incident particles and the TPC, Time of Flight (ToF), multicell Cherenkov (Ckov) and Ring Imaging Cherenkov (RICH) detectors for identifying secondary particles [2]. The TPC identifies the particles up to $1 \mathrm{GeV} / \mathrm{c}$, the ToF up to $2 \mathrm{GeV} / \mathrm{c}$, the Ckov up to 17 $\mathrm{GeV} / \mathrm{c}$ and the RICH up to $120 \mathrm{GeV} / \mathrm{c}$.

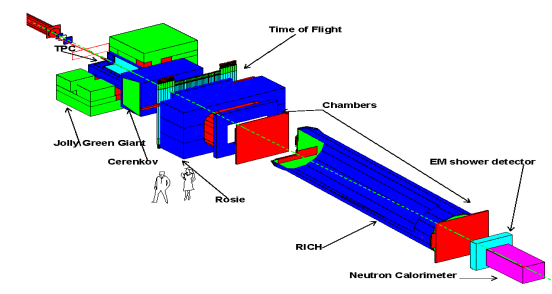

FIGURE 1. Layout of the MIPP experiment.

\section{MOTIVATION}

Previous experiments used single-arm spectrometers, giving only single $\left(\mathrm{p}, \mathrm{p}_{\mathrm{T}}\right)$ flux measurements. As a result, hadronic production models like GEANT4, MARS and FLUKA have limited amount of data to be compared with and in some cases they give significantly different results. The MIPP is an open-geometry spectrometer giving complete angular coverage in $\left(\mathrm{p}_{\mathrm{Z}}, \mathrm{p}_{\mathrm{T}}\right)$ space.

Scarcity of hadronic production data affects every atmospheric and accelerator-based neutrino experiment. The uncertainties in particle production models largely cancel in comparison of the near and far detector data in case of a two-detector neutrino experiment, but calculation of neutrino flux with various hadronic production models differ by up to $30 \%$. As an example, in case of Main Injector Oscillation Search (MINOS) experiment, the near detector sees a line source of neutrinos and the far detector sees a point source of neutrinos, so, these uncertainties do not cancel completely, and the near/far 

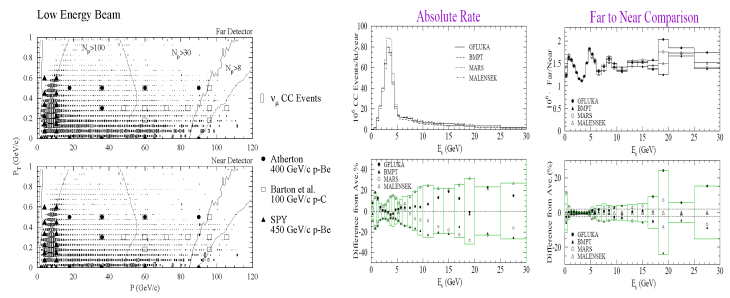

FIGURE 2. Left- The near and far detector neutrino spectra as function of longitudinal and transverse momentum. The discrete points show the available data from previous hadronic production measurements using single arm spectrometers. Right- Uncertainties in neutrino flux calculation in NuMI beam.

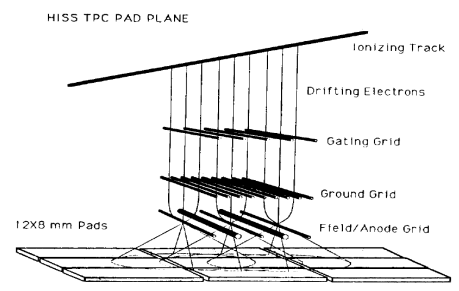

FIGURE 3. Schematic view of the TPC pad plane.

detector comparison depends on the hadron production model as shown in the Figure 2.

Neutrino flux problems in MINOS, MiniBooNE, $\mathrm{K} 2 \mathrm{~K}, \mathrm{~T} 2 \mathrm{~K}$, NovA, MINER $v \mathrm{~A}$ can be reduced to one problem: the current status of insufficient knowledge of the strong interactions. Better understanding of hadronic production can minimize the systematic error in neutrino flux predictions.

\section{THE MIPP DETECTORS}

Time Projection Chamber: The TPC is the centerpiece of the MIPP spectrometer. The TPC is operated with $\mathrm{P} 10\left(90 \% \mathrm{Ar}, 10 \% \mathrm{CH}_{4}\right)$ gas. Electrons produced in the ionization of the gas caused by charged particles drift down in a uniform vertical electric field of $125 \mathrm{~V} / \mathrm{cm}$. The image charge on the pad plane $4 \mathrm{~mm}$ below the anode wires is recorded by the electronics. The pad plane is divided into 128 pad rows along the $\mathrm{z}$-axis with 120 , $0.8 \times 1.2 \mathrm{~cm}$ pads per row along the $\mathrm{x}$-axis. The schematic view of the TPC pad plane with anode and ground wires is shown in Figure 3. The pads give position in $\mathrm{x}$ and $\mathrm{z}$ direction and drift time measurement gives the y coordinate.

Ring Imaging Cherenkov Detector: The $10 \mathrm{~m}$ long radiator volume is filled with carbon dioxide to a total pressure of 1.03 atmosphere. Sixteen hexagonal spherical focusing mirrors mounted at the downstream end re-

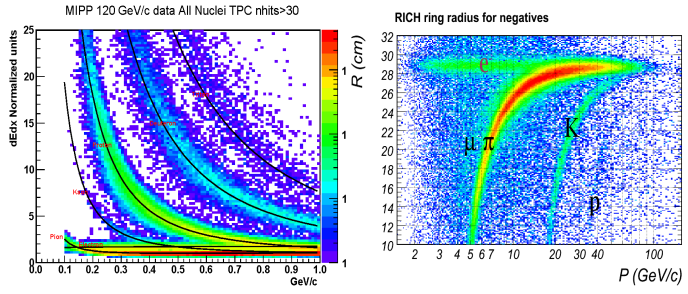

FIGURE 4. Left- TPC provides the particle separation in momentum range $0.1-1 \mathrm{GeV} / \mathrm{c}$ and the $\mathrm{dE} / \mathrm{dx}$ resolution is $12 \%$. Right- RICH ring radii distributions give clean separation of $\pi, \mathrm{K}$ and $\mathrm{p}$ above $\sim 20 \mathrm{GeV} / \mathrm{c}$ and e/ $\mu / \pi$ up to $12 \mathrm{GeV} / \mathrm{c}$.

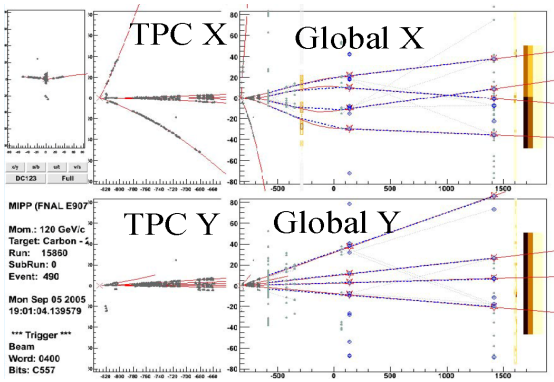

FIGURE 5. Reconstructed $\mathrm{p}+\mathrm{C} 120 \mathrm{GeV} / \mathrm{c}$ event

flect Cherenkov light onto an array of 1 inch PMTs which allows the reconstruction of rings. The radii of the rings are measured.

The MIPP TPC identifies the particle type using the $\mathrm{dE} / \mathrm{dx}$ calculation and the RICH detector identifies the particle type by measuring the ring radius. In Figure 4, the left plot shows the $\mathrm{dE} / \mathrm{dx}$ as a function of momentum of the particle in the TPC and the right plot shows the ring radii as a function of the particle momentum in RICH.

\section{TRACK AND VERTEX RECONSTRUCTION}

The track reconstruction is divided into two parts: 1) TPC tracks are determined. 2) TPC tracks are matched to wire chamber hits to form global tracks. In Figure 5, a reconstructed $\mathrm{p}+\mathrm{C} 120 \mathrm{GeV} / \mathrm{c}$ event is shown.

There are two steps to vertexing: 1) Vertex finding is done with iterative algorithm. 2) All tracks from each vertex are refit with the constraint of originating at the vertex. It uses track templates. The vertex resolution along the $\mathrm{Z}$-axis is $\sim 6 \mathrm{~mm}$ and along the $\mathrm{X}$ and $\mathrm{Y}$-axis is $<1 \mathrm{~mm}$ 

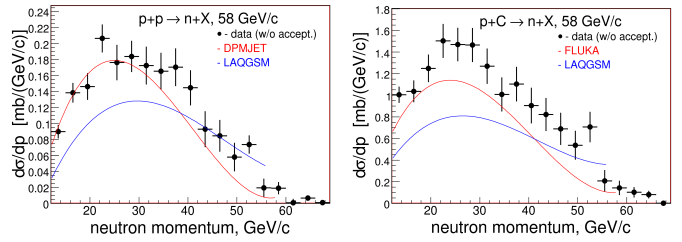

FIGURE 6. Measured cross sections from this experiment compared with predictions from Monte Carlos.
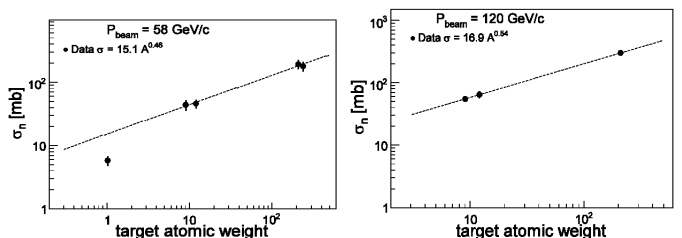

FIGURE 7. Neutron production cross sections from MIPP experiment as a function of target atomic weight $\mathrm{A}$.

\section{RECENT RESULTS}

Forward Neutron Inclusive Cross sections: The MIPP has measured cross sections for forward neutron production from a variety of targets using proton beam momenta of 58, 84, and $120 \mathrm{GeV} / \mathrm{c}$ [3]. The cross sections show reasonable agreement with FLUKA and DPMJET Monte Carlos. Figure 6 shows the comparison of measured cross sections with predictions from the Monte Carlos and Figure 7 shows the cross sections as a function of target atomic weight $\mathrm{A}$.

Preliminary Results From MINOS Target Data Analysis: The MIPP has obtained data on particle production using $120 \mathrm{GeV} / \mathrm{c}$ protons from the Main Injector impinging on the MINOS target. It has determined the rates for inclusive production of $\mathrm{e}^{ \pm}, \pi^{ \pm}, \mathrm{K}^{ \pm}, \mathrm{p}$ and $\overline{\mathrm{p}}$ particles as a function of longitudinal and transverse momentum of the final state particle [4]. This analysis uses the Bayes' theorem algorithms to identify whether the particle is electron, pion, kaon or proton. So, there are four hypotheses $\mathrm{e}, \pi, \mathrm{K}$ and $\mathrm{p}$. The probability of each hypothesis is iteratively determined. Figure 8 shows the comparison of GlobalPid spectra with the MCTRUTH in the Monte Carlo sample. GlobalPid agrees well with the MCTRUTH. Figure 9 shows the comparison of positive and negative spectra for the four particle types in the data.

\section{FUTURE ANALYSES}

The MINOS target analysis is very close to completion. The production cross section calculations for 20,58 , $85 \mathrm{GeV} / \mathrm{c} \pi, \mathrm{K}$ and $\mathrm{p}$ on $\mathrm{LH}_{2}$ and also thin targets $\mathrm{C}, \mathrm{Be}$,
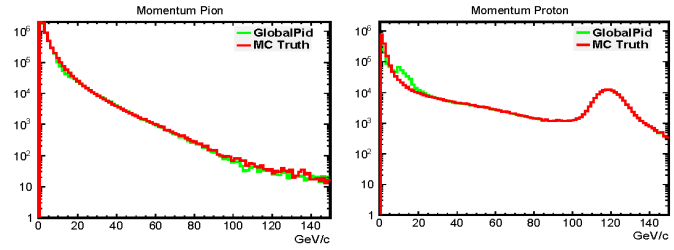

FIGURE 8. Comparison of the GlobalPid spectra (green) at the end of 15 iterations with MCTRUTH (red) in the Monte Carlo sample for both charges for pions (left) and for protons (right).
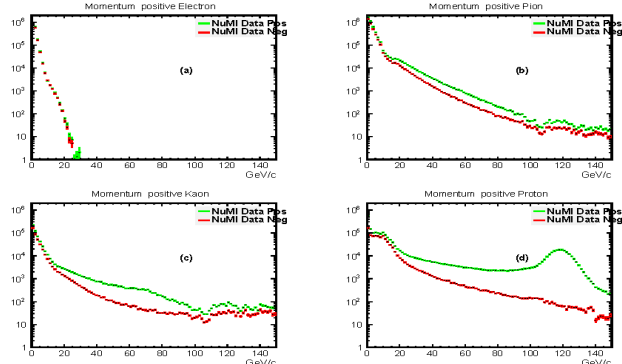

FIGURE 9. Comparison of the data positive (green) and negative (red) spectra (a) for electrons (b) for pions (c) for kaons and (d) for protons.

$\mathrm{Bi}$ and $\mathrm{U}$ are in progress. The future analyses include calculation of neutral Kaon production cross sections, testing the "Scaling Law" of inclusive cross sections, providing data for studies of non-perturbative QCD, investigating light meson spectroscopy and missing baryon resonances.

\section{ACKNOWLEDGMENTS}

The author is grateful to the organizers of NuFact10 conference for providing the opportunity to present this work. This work is supported by Fermilab under IndiaFermilab Neutrino Collaboration.

\section{REFERENCES}

1. Rajendran Raja, The Main Injector Particle Physics Experiment (MIPP FNAL E-907) at Fermilab - status and plans, arxiv:hep-ex/0612007 (2006).

2. Holger Meyer, The Main Injector Particle Production experiment (MIPP) at Fermilab, J. Phys.:Conf. Ser.69:012025 (2007).

3. T. S. Nigmanov et. al., Forward Neutron Production at the Fermilab Main Injector, arxiv:hep-ex/1010.6291 (2010) (To be published in Phys. Rev. D).

4. U. Akgun et. al., Measurement of particle production on the NuMI target with a $120 \mathrm{GeV} / \mathrm{c}$ proton beam in the MIPP experiment (To be submitted in Phys. Rev. D). 